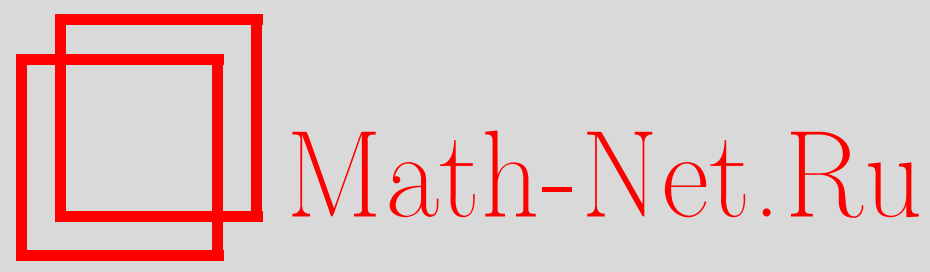

М. Э. Михайлов, Дополнительные признаки неразложимости конечных псевдометрических пространств, Матем. заметки, 1998, том 63, выпуск 3, 421-424

DOI: https://doi.org/10.4213/mzm1298

Использование Общероссийского математического портала Math-Net.Ru подразумевает, что вы прочитали и согласны с пользовательским соглашением http://www.mathnet.ru/rus/agreement

Параметры загрузки:

IP : 54.162 .127 .20

26 апреля 2023 г., $17: 02: 54$ 


\section{ДОПОЛНИТЕЛЬНЫЕ ПРИЗНАКИ НЕРАЗЛОЖИМОСТИ КОНЕЧНЫХ ПСЕВДОМЕТРИЧЕСКИХ ПРОСТРАНСТВ}

\section{М.Э. Михайлов}

Продолжено исследование признаков конечных (состоящих из конечного числа точек) неразложимых псевдометрических пространств, которые нельзя разложить в сумму любым иньм способом, кроме деления всех расстояний в одинаковой пропорции. Доказано, что неразложимость сохраняется, если в представляющем пространство графе две несвязанные ребром вершины соединить добавочной простой цепью, которая является копией соединяющего эти вершины кратчайшего маршрута, но присоединена противоположными концами. Доказано также, что неразложимы пространства, изображаемые графами $K_{m, n}(m \geqslant 2, n \geqslant 3)$ с ребрами равной длины.

Библиография: 3 названия.

В предыдущей работе [1] было введено понятие неразложимого псевдометрического пространства: это псевдометрическое пространство, которое нельзя разложить в сумму любым иным способом, кроме деления всех расстояний в одинаковой пропорции. Была также выведена процедура проверки на неразложимость любого конечного (состоящего из конечного числа точек) псевдометрического пространства, которая заключается в составлении и решении так называемой проверочной системы уравнений, и установлены некоторые признаки разложимости и неразложимости. В данной работе выводятся два новых признака неразложимости в дополнение к ранее полученным.

Будем пользоваться способом представления псевдометрических пространств в виде графов, который применялся в предыдущей работе [1] и в основном восходит к Зыкову [2]. Ребра графа представляют все те расстояния $r(a, b)$, которые не являются суммой $r(a, b)=r(a, c)+r(b, c)$, где $c$-любая третья точка. Расстояния проставляются рядом с ребрами, если они не равны 1. Расстояние, не представленное ребром, равно кратчайшему маршруту, соединяющему конщевые точки [2]. В псевдометрическом пространстве, в отличие от метрического, возможны группы точек с нулевыми расстояниями между ними (кратные вершины). Такая группа рассматривается как одна вершина графа и в данной работе особо не обозначается.

ОПРЕДЕЛЕНИЕ. Простой иепью графа назьвают цеп без повторяющихся вершин и ребер [3]. Назовем свободной иепью простую цепь, состоящую более чем из одного ребра и связанную с остальной частью графа лишш крайними вершинами. Рефлексом (отражением) простой цепи назовем свободную цепь, которая является ее копией и связана с ней общими крайними вершинами. Если обе цепи соединены аналогичными вершинами, будем назьвать рефлекс прямым, если противоположными - обратным. Сказанное пояснено на рис. 1. 
a)

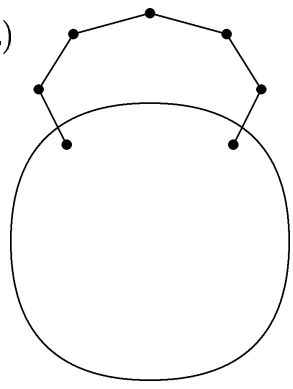

б)

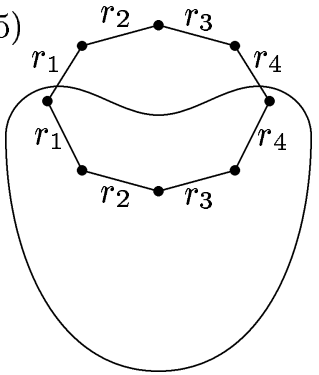

B)

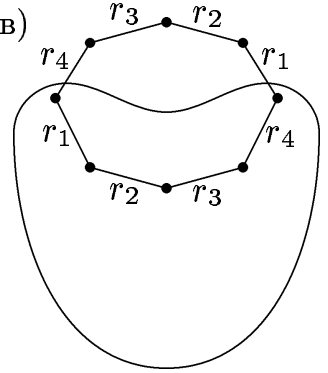

Рис. 1. Криволинейная фигура - граф, цепь за ее пределами а) свободная цепь, б) прямой рефлекс, в) обратньй рефлекс

ТЕОРемА 1. Если в неразложсимом псевдометрическом пространстве к произвольному кратчайшему маршруту, состоящему более чем из одного ребра, присоединить его обратный рефлекс, то неразлохимость сохранится.

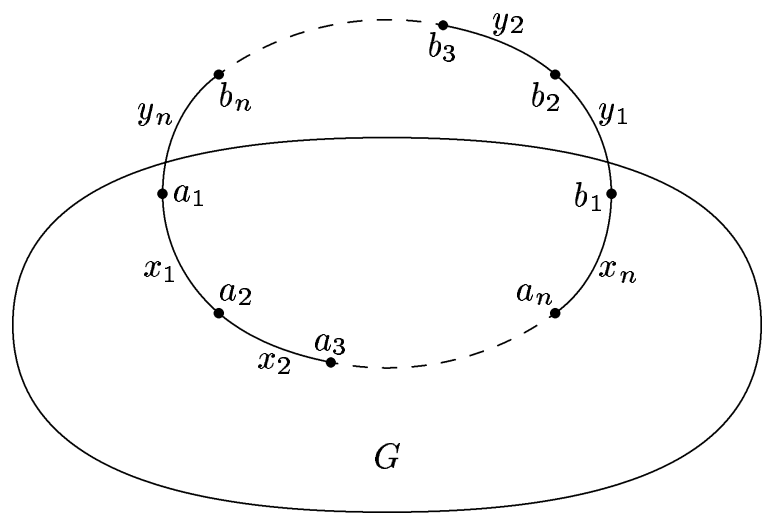

Р ис. 2. Криволинейная фигура - пространство $G$, та же фигура с обратньм рефлексом $b_{1} b_{2} \cdots b_{n} a_{1}$ кратчайшего маршрута $a_{1} a_{2} \cdots a_{n} b_{1}-$ пространство $G r$

ДокАЗАТЕЛЬСтво. Обозначим исходное пространство через $G$, а пространство с обратным рефлексом - через $G r$ (рис. 2). Пусть $G$ неразложимо. С присоединением обратного рефлекса проверочная система уравнений (см. [1]) для части $G$ не изменится, так как ни для одной пары вершин из $G$ не появится более короткого маршрута. Если бы он появился, то мог бы проходить только через цеп $y_{1} \cdots y_{n}$. Но тогда равную длину должен иметь и маршрут, проходяший через цепь $x_{1} \cdots x_{n}$. Таким образом, в проверочной системе все стороны из $G$ определятся однозначно (с точностью до постоянного коэффищиента), поскольку пространство $G$ неразложимо. С присоединением обратного рефлекса в системе появятся новые уравнения, связанные с циклом $y_{1} \cdots y_{n} x_{1} \cdots x_{n}$. Кратчайшие маршруты между вершинами цикла должны лежать в цикле. Пусть, например, существует маршрут из $b_{2}$ в $a_{2}$ меньший, чем $y_{1} x_{n} \cdots x_{2}$ или $y_{2} \cdots y_{n} x_{1}$. Каков бы он ни был, он должен проходить через $a_{1}$ или $b_{1}$. Но кратчайший маршрут (или один из них) от $a_{1}$ или $b_{1}$ до $a_{2}$ проходит по цепи $a_{1} a_{2} \cdots a_{n}$. 
Следовательно, получаем дополнительные уравнения:

$$
\begin{aligned}
& x_{1}+x_{2}+\cdots+x_{n}=y_{1}+y_{2}+\cdots+y_{n}, \\
& x_{2}+\cdots+x_{n}+y_{1}=y_{2}+\cdots+y_{n}+x_{1} \text {, } \\
& x_{n}+y_{1}+\cdots+y_{n-1}=y_{n}+x_{1}+\cdots+x_{n-1} \text {. }
\end{aligned}
$$

Решив систему, получим $y_{1}=x_{1}, y_{2}=x_{2}, \ldots, y_{n}=x_{n}$. Таким образом, все ребра графа $G r$ однозначно (с точностью до постоянного коэффициента) определяются его проверочной системой. Значит, пространство $G r$ неразложимо. Теорема доказана.

СлЕДСТВИЕ 1. К любому кратчайшему маршруту можно присоединить сколько угодно обратных рефлексов с сохранением неразложимости.

СлЕДСТВИЕ 2. К любому кратчайшему маршруту можно присоединить сколько угодно прямых и обратных рефлексов с сохранением неразложсимости, если среди них есть хотя бы один обратный.
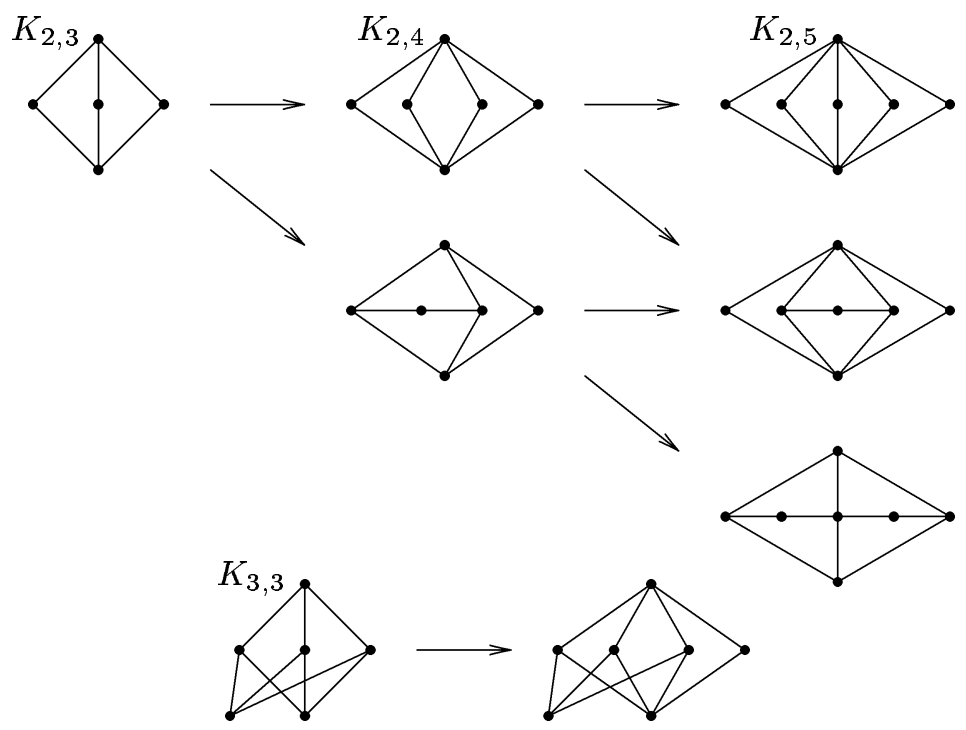

Р ис. 3. Присоединение рефлексов к неразложимым пространствам. Все ребра графов имеют длину 1

На рис. 3 даны примеры получения одних неразложимых пространств из других путем присоединения рефлексов. В данном случае все эти рефлексы одновременно и прямые и обратные.

Следуюшие графы называются полными двудольными (бихроматическими): вершины в них разбиты на две групшы, каждая пара вершин из разных групп связана ребром, других ребер нет. Обозначаются такие графы через $K_{m, n}$, где $m, n$ - число вершин в группах [2]. На рис. 3 указаны пространства, изображаемые графами $K_{2,3}, K_{2,4}$, $K_{3,3}, K_{2,5}$. Все их ребра имеют длину 1 , поэтому равны 1 все межгрупповые расстояния, а все внутригрупповые расстояния равны 2. 
ТЕорема 2. Псевдометрические пространства, изображкаемые графами $K_{m, n}$ $(m \geqslant 2, n \geqslant 3)$ с ребрами равной длинь, неразложимы.

ДокАЗАТЕльСтво. Примем для определенности, что длина ребер равна 1. Пусть существует удовлетворяющее условиям теоремы неразложимое пространство $G$ с числом вершин $m$ в групе $a$ и числом вершин $n$ в группе $b$. Добавив вершину $c$ к группе $a$, получим пространство $G c$ (рис. 4 ). В графе $G$ все расстояния не превышают 2 , так что добавление вершины $c$ не приведет к сокрашению расстояний, и ребра в части $G$ будут по-прежнему однозначно определяться проверочной системой. Поэтому ребра $a_{i} b_{j}$ считаем вычисленными. Рассчитаем теперь ребра $x_{1} \cdots x_{n}$. Любая пара ребер $x_{i}, x_{j}$ входит в простой цикл $c b_{i} a_{k} b_{j}$, что дает уравнения $x_{i}+1=x_{j}+1$ и $x_{i}+x_{j}=2$, из которых следует $x_{i}=x_{j}=1$. Таким образом, все ребра $x_{1} \cdots x_{n}$ однозначно определяются. Следовательно, если $G$ неразложимо, то неразложимо и $G c$.

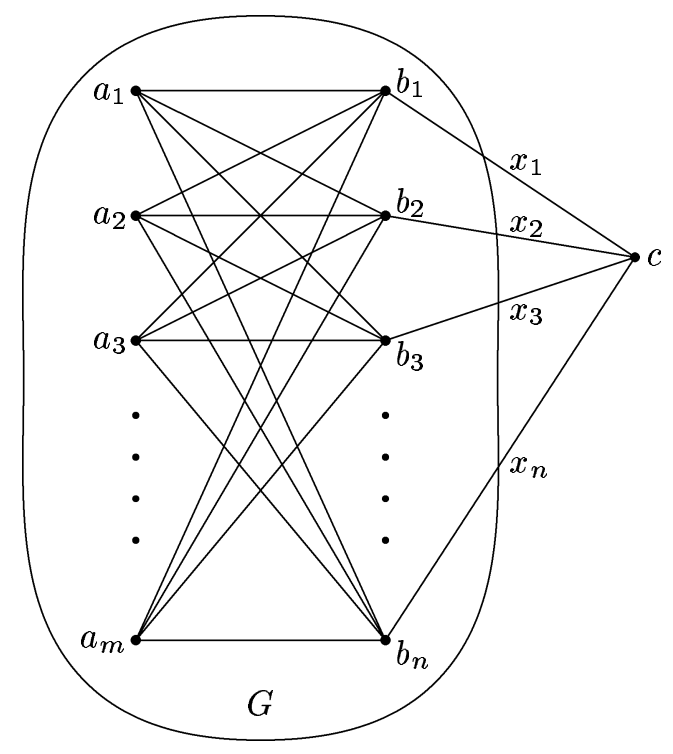

Рис. 4. Криволинейная фигура - пространство $G$, та же фигура с точкой $c$-пространство $G c$

Пространство, изображаемое графом $K_{2,3},-$ минимальное неразложимое пространство подобного вида [1]. Все другие пространства, удовлетворяюшие условиям теоремы, можно получить из $K_{2,3}$ последовательным присоединением вершин и умножением расстояний на постоянный коэффициент. Следовательно, все они неразложимы. Теорема доказана.

\section{СПИСОК ЦИТИРОВАННОЙ ЛИТЕРАТУРЫ}

[1] Михайлов М. Э. Разложениеконечных псевдометрических пространств // Матем. заметки. 1998. Т. 63. № 2. C. 225-234.

[2] Зыков А. А. Основы теории графов. М.: Наука, 1987.

[3] Оре О. Теория графов. М.: Наука, 1980.

Институт генетики АН Республики Молдова, г. Кишинев 